This is the final peer-reviewed accepted manuscript of:

Lucia Romani: Interpolating m-refinable functions with compact support: The second generation class, Applied Mathematics and Computation, Volume 361, 2019, Pages 735-746

The final published version is available online at:

https://doi.org/10.1016/i.amc.2019.06.018

Rights / License:

The terms and conditions for the reuse of this version of the manuscript are specified in the publishing policy. For all terms of use and more information see the publisher's website.

This item was downloaded from IRIS Università di Bologna (https://cris.unibo.it/)

When citing, please refer to the published version. 


\title{
Interpolating $m$-refinable functions with compact support: the second generation class
}

\author{
Lucia Romani ${ }^{\mathrm{a}, *}$ \\ ${ }^{a}$ Dipartimento di Matematica, Alma Mater Studiorum Università di Bologna, \\ Piazza di Porta San Donato 5, 40126 Bologna, Italy
}

\begin{abstract}
We present an algorithm for the construction of a new class of compactly supported interpolating refinable functions that we call the second generation class since, contrary to the existing class, is associated to subdivision schemes with an even-symmetric mask that does not contain the submask $\{0 \ldots, 0,1,0, \ldots 0\}$. As application examples of the proposed algorithm we present interpolating 4-refinable functions that are generated by parameter-dependent, even-symmetric quaternary schemes never considered in the literature so far.
\end{abstract}

Keywords: Subdivision; Interpolation; Even-symmetric mask; Arity $m$; Refinable function

\section{Introduction and purpose of the work}

Compactly supported refinable functions for interpolation are of interest in several fields of application including sampling theory, signal processing, computer graphics and geometric modelling.

Throughout this paper, $m$ denotes a dilation factor (that is an integer greater than 1) and with symmetric, $m$-refinable function of compact support we refer to any function $\varphi: \mathbb{R} \rightarrow \mathbb{R}$ with $\operatorname{supp}(\varphi)=\left[0, \frac{M}{m-1}\right]$, $M \in \mathbb{N}$, that satisfies both the symmetry condition

$$
\varphi(x)=\varphi\left(\frac{M}{m-1}-x\right), \quad \forall x \in \mathbb{R}
$$

and the refinement equation

$$
\varphi(x)=\sum_{j=0}^{M} a_{j} \varphi(m x-j), \quad \forall x \in \mathbb{R} .
$$

The coefficients in 1.2 are the entries of a finitely supported sequence $\boldsymbol{a}=\left\{a_{j} \in \mathbb{R}, j \in \mathbb{Z}\right\}$ that fulfills

$$
a_{0}, a_{M} \neq 0 \text { and } a_{j}=0 \text { for } j<0 \text { and } j>M,
$$

and is usually called the subdivision mask of the $m$-refinable function $\varphi$.

The class of compactly supported functions that, in addition to 11.1 and $(1.2)$, satisfies

$$
\varphi\left(n+\frac{\tau_{\boldsymbol{a}}}{m-1}\right)=\delta_{0, n}, \quad n \in \mathbb{Z} \quad \text { with } \quad \tau_{\boldsymbol{a}}=\frac{M}{2},
$$

\footnotetext{
* Corresponding author

Email address: lucia.romani@unibo.it (Lucia Romani)
} 
is called interpolating. Here, $\delta$ denotes the Kronecker delta while the real number $\tau_{\boldsymbol{a}}$ is the so-called shift parameter. In case of a subdivision mask $\boldsymbol{a}$ satisfying $(1.3), \tau_{\boldsymbol{a}}$ is computed from the degree- $M$ polynomial

$$
A(z)=\sum_{j=0}^{M} a_{j} z^{j}, \quad z \in \mathbb{C} \backslash\{0\}
$$

as $\tau_{\boldsymbol{a}}=\frac{A^{\prime}(1)}{m}$ (see, e.g., [5]) where $A^{\prime}(z)$ denotes the first derivative of $A(z)$. In particular, in [5] it is proven that, in case of a subdivision scheme with odd-symmetry (see Definition 2.2), $\tau_{\boldsymbol{a}} \in \mathbb{Z}$; conversely, in case of even-symmetry (see Definition 2.3 ,,$\tau_{\boldsymbol{a}} \in \frac{\mathbb{Z}}{2} \backslash \mathbb{Z}$.

Symmetric, compactly supported interpolating $m$-refinable functions that we can find in the literature always fulfill equation (1.4) in the case $\tau_{\boldsymbol{a}} \in \mathbb{Z}$, i.e., are associated with an odd-symmetric subdivision mask $\boldsymbol{a}$ satisfying (1.3) for an even integer $M$ (see, e.g., 1, 2, 8, 11, 15, 16, 18, 23, 24). In addition, for all of them the odd-symmetric subdivision mask $\boldsymbol{a}$ is such that

$$
a_{m j+\frac{M}{2}}=\delta_{0, j}, \quad j \in \mathbb{Z} \cap\left[-\left\lfloor\frac{M}{2 m}\right\rfloor,\left\lfloor\frac{M}{2 m}\right\rfloor\right],
$$

i.e., it contains the submask $\{0 \ldots, 0,1,0, \ldots 0\}$.

The goal of this work is to introduce a novel class of subdivision schemes that, differently from the existing ones, are associated with symmetric, compactly supported interpolating $m$-refinable functions satisfying equation (1.4) with $\tau_{\boldsymbol{a}} \in \frac{\mathbb{Z}}{2} \backslash \mathbb{Z}$. More precisely, the subdivision schemes gathered in the new class are defined via an even-symmetric subdivision mask that satisfies 1.3 for an odd integer $M$. Since condition 1.6. is not fulfilled, the new class is called the second generation class of interpolating schemes.

In the remainder of the paper, we first recall some background notions about subdivision schemes and refinable functions (Section 2), then we present our main result (Section 3). Closing remarks are provided in Section 4

\section{Background notions on subdivision schemes}

Subdivision schemes of arity $m$ are iterative methods that, starting from the initial sequence of points $\boldsymbol{p}^{0}=\left\{p_{j}^{0}, j \in \mathbb{Z}\right\}$, generate finer and finer sequences of points $\boldsymbol{p}^{k+1}$ by using the subdivision rules

$$
p_{m i+h}^{k+1}=\sum_{j \in \mathbb{Z}} a_{m(i-j)+h} p_{j}^{k}, \quad h=0, \ldots, m-1,
$$

at each refinement level $k \in \mathbb{N}_{0}$ (with $\mathbb{N}_{0}$ the nonnegative set of integers). If 2.1 are suitably defined, when $k$ approaches infinity the sequence of piecewise linear functions which interpolate the data at level $k$ converges uniformly to a continuous (and possibly smooth) limit function (for the theoretical results concerning the analysis of convergence and smoothness of subdivision schemes, we refer the reader to [10, 12, where the preparatory binary case is extensively discussed).

Definition 2.1. The limit function generated by a convergent subdivision scheme starting from the data $p_{j}^{0}=\delta_{0, j}, j \in \mathbb{Z}$, is called basic limit function and denoted by $\varphi$.

The basic limit function of a subdivision scheme $\mathcal{S}_{\boldsymbol{a}}$ of arity $m$ and subdivision mask $\boldsymbol{a}=\left\{a_{0}, \ldots, a_{M}\right\}$ has support $\left[0, \frac{M}{m-1}\right]$ (see, e.g., [5, page 415] or [12, page 78]) and satisfies the refinement equation in 1.2]. Thus, the arity is also equivalently called dilation.

Definition 2.2. The arity-m subdivision scheme $\mathcal{S}_{\boldsymbol{a}}$ with symbol 1.5 is called odd-symmetric if $M$ is an even positive integer and $a_{i}=a_{M-i}$ for all $i=0, \ldots, \frac{M}{2}$, or equivalently, $A(z) z^{-\frac{M}{2}}=A\left(z^{-1}\right) z^{\frac{M}{2}}$.

Definition 2.3. The arity-m subdivision scheme $\mathcal{S}_{\boldsymbol{a}}$ with symbol 1.5$)$ is called even-symmetric if $M$ is an odd positive integer and $a_{i}=a_{M-i}$ for all $i=0, \ldots, \frac{M-1}{2}$, or equivalently, $A(z) z^{-\frac{M-1}{2}}=A\left(z^{-1}\right) z^{\frac{M+1}{2}}$. 
Definition 2.4. A subdivision scheme is called interpolating if it is convergent and, for any given sequence $\boldsymbol{p}^{0}$, produces a limit function that passes through all the vertices of $\boldsymbol{p}^{0}$.

The basic limit function of an arity- $m$ even/odd symmetric interpolating scheme whose subdivision mask satisfies (1.3), fulfills 1.4 . We conclude this preliminary section by additionally observing that, under the assumption that the subdivision mask $\boldsymbol{a}$ satisfies $(1.3)$ and is either even or odd symmetric, the subdivision symbol of $\mathcal{S}_{a}$ is the degree- $M$ polynomial $A(z)$ in 1.5$)$ and its first derivative fulfills $A^{\prime}(1)=\frac{m M}{2}[5$, Remark 5.8]. Thus, the conditions to be satisfied by $A(z)$ for achieving the property of reproduction of the space $\Pi_{d}$ of polynomials up to degree $d$ (see [5, Theorem 4.3]) can be formulated in terms of the same shift parameter $\tau_{\boldsymbol{a}}=\frac{M}{2}$ in the unified form

$$
A(1)=m, \quad A^{(i)}(1)=m \prod_{h=0}^{i-1}\left(\tau_{\boldsymbol{a}}-h\right) \text { for all } i=1, \ldots, d, \quad A^{(i)}\left(e^{\frac{2 \pi \mathrm{i}}{m} j}\right)=0 \text { for all } \begin{gathered}
j=1, \ldots, m-1 \\
\text { and } \\
i=0, \ldots, d .
\end{gathered}
$$

Remark 2.5. Note that when (1.6) is satisfied, then the subdivision rules (2.1) have the property of naturally retaining the initial data $p_{j}^{0}, j \in \mathbb{Z}$ at each iteration. In this way, the initial data will become points of the limit function itself, thus endowing it with the interpolation property. However, interpolation can be achieved even without requiring the fulfilment of (1.6). In the latter case, although the given data $p_{j}^{0}, j \in \mathbb{Z}$ are still points of the limit function, the sequences of points $\boldsymbol{p}^{k}$ generated during the iterations will not lie on the limit function (see, e.g., Figure 4 for an application example to sequences of points in $\mathbb{R}^{2}$ ).

\section{Main result}

Since we are interested in even-symmetric subdivision schemes $\mathcal{S}_{\boldsymbol{a}}$ with $\tau_{\boldsymbol{a}} \in \frac{\mathbb{Z}}{2} \backslash \mathbb{Z}$, we focus on arity-m, convergent subdivision schemes defined by a subdivision mask $\boldsymbol{a}$ as in 1.3 where $M$ is odd and multiple of $m-1$, the symmetry condition $a_{j}=a_{M-j}, j=0, \ldots, M$ holds true and the shift parameter is $\tau_{\boldsymbol{a}}=\frac{M}{2}$. Under such assumptions, the basic limit function $\varphi$ of $\mathcal{S}_{a}$ satisfies

I) $\operatorname{supp}(\varphi)=\left[0, \frac{M}{m-1}\right]$ and $\varphi(x)=\varphi\left(2 \frac{\tau_{a}}{m-1}-x\right)$ for all $x \in \mathbb{R}$.

Property $I$ ) means that $\varphi$ is compactly supported and symmetric about the point $\frac{\tau_{a}}{m-1}$.

In our work we additionally require that $\varphi$ satisfies the interpolation condition

$$
\text { II) } \varphi\left(n+\frac{\tau_{a}}{m-1}\right)=\delta_{0, n}, n \in \mathbb{Z} .
$$

More precisely, property $I I)$ implies that the values of the $\delta$ sequence are attained at $\frac{\mathbb{Z}}{2} \backslash \mathbb{Z}$ since $\frac{\tau_{a}}{m-1} \in \frac{\mathbb{Z}}{2} \backslash \mathbb{Z}$. In fact, in light of the fact that $M$ is odd, we can observe that by assuming $m$ even and such that $m-1$ divides $M$, then $\frac{M}{m-1}$ turns out to be an odd integer and consequently $\frac{\tau_{a}}{m-1}=\frac{M}{2(m-1)} \in \frac{\mathbb{Z}}{2} \backslash \mathbb{Z}$.

Proposition 3.1. The integer translates of a basic limit function $\varphi$ that satisfies property II) are linearly independent.

Proof. Let $F:=\sum_{j \in \mathbb{Z}} c_{j} \varphi(\cdot-j)$ and suppose that $F \equiv 0$. In light of II), evaluation of $F$ at $n+\frac{\tau_{a}}{m-1}, n \in \mathbb{Z}$ implies that $\boldsymbol{c}$ is the zero sequence, so proving the claimed result.

Proposition 3.2. The integer translates of a basic limit function $\varphi$ that satisfies properties I) and II) are $\ell^{\infty}$-stable, namely fulfill the stability condition

$$
K_{1}\|\boldsymbol{c}\|_{\infty} \leq\left\|\sum_{j \in \mathbb{Z}} c_{j} \varphi(\cdot-j)\right\|_{\infty} \leq K_{2}\|\boldsymbol{c}\|_{\infty}, \quad \boldsymbol{c} \in \ell^{\infty}(\mathbb{Z}), \quad 0<K_{1} \leq K_{2}<\infty .
$$


Proof. Let $\frac{\tau_{a}}{m-1} \in \Omega \subset \mathbb{R}$. In light of II),

$$
\left\|\left|\sum_{j \in \mathbb{Z}} c_{j} \varphi(\cdot-j)\left\|_{\infty}=\sup _{x \in \mathbb{R}}\left|\sum_{j \in \mathbb{Z}} c_{j} \varphi(x-j)\right| \geq \sup _{x \in \Omega}\left|\sum_{j \in \mathbb{Z}} c_{j} \varphi(x-j)\right|=\sup _{j \in \mathbb{Z}}\left|c_{j}\right|=\right\| \boldsymbol{c} \|_{\infty},\right.\right.
$$

which yields $K_{1}=1$. To show the existence of the upper bound in 3.1 we can write, for all $x \in \mathbb{R}$,

$$
\left\|\sum_{j \in \mathbb{Z}} c_{j} \varphi(\cdot-j)\right\|_{\infty} \leq \sup _{x \in \mathbb{R}} \sum_{j \in \mathbb{Z}}\left|c_{j}\right||\varphi(x-j)| \leq\|\boldsymbol{c}\|_{\infty} \sup _{x \in \mathbb{R}} \sum_{j \in \mathbb{Z}}|\varphi(x-j)| \leq\|\boldsymbol{c}\|_{\infty}\|\varphi\|_{\infty}\lceil|\operatorname{supp}(\varphi)|\rceil .
$$

Thus, $K_{2}=\|\varphi\|_{\infty}\lceil|\operatorname{supp}(\varphi)|\rceil$ where $\lceil|\operatorname{supp}(\varphi)|\rceil=\left\lceil\frac{M}{m-1}\right\rceil$ in light of the fact that $\operatorname{supp}(\varphi)=\left[0, \frac{M}{m-1}\right]$.

3.1. Even-symmetric, interpolating m-refinable functions with compact support: the second generation class

Assuming that $\varphi$ is generated by the subdivision mask $\boldsymbol{a}$ in (1.3), we can express its values at half-integers by means of the following relation provided by its $m$-refinability property

$$
\varphi\left(\frac{i}{2}\right)=\sum_{j \in \mathbb{Z}} a_{j} \varphi\left(\frac{m}{2} i-j\right), \quad i \in \mathbb{Z} .
$$

Equivalently, 3.2 can be reformulated as a bi-infinite linear system of the form

$$
\mathrm{X} a=\mathrm{y}
$$

where

$$
y_{i}=\varphi\left(\frac{i}{2}\right), \quad i \in \mathbb{Z}
$$

is the $i$-th entry of the right-hand side of 3.3 and

$$
X_{i, j}=\varphi\left(\frac{m}{2} i-j\right), \quad i, j \in \mathbb{Z}
$$

is the $(i, j)$-entry of the bi-infinite matrix $\mathbf{X}$ which turns out to be banded due to the compact support of $\varphi$. In order to construct an $m$-refinable function $\varphi$ that satisfies the property in $\sqrt{1.4}$ in the case $\tau_{\boldsymbol{a}} \in \frac{\mathbb{Z}}{2} \backslash \mathbb{Z}$, we assume $m$ even and $M$ odd such that $m-1$ divides $M$. As a consequence, $\operatorname{supp}(\varphi)=[0, S]$ with $S=\frac{M}{m-1} \in 2 \mathbb{Z}+1$ and we require that

$$
\left\{\varphi\left(\frac{i}{2}\right)\right\}_{i=1, \ldots, 2 S-1}= \begin{cases}\delta_{0, i-S}, & i \in 2 \mathbb{Z}+1 \cap(0,2 S) \\ v_{\frac{i}{2}}, & i \in 2 \mathbb{Z} \cap(0,2 S)\end{cases}
$$

where $\boldsymbol{v}=\left\{v_{1}, v_{2}, \ldots, v_{S-1}\right\}$ is a vector with $S-1$ input values assigned by the user and satisfying $\sum_{i=1}^{S-1} v_{i}=$ 1 since convergence of the subdivision scheme $\mathcal{S}_{\boldsymbol{a}}$ requires $\varphi$ to enjoy the property of reproducing constants. Since $S \in 2 \mathbb{Z}+1$ then $S-1 \in 2 \mathbb{Z}$ and $\boldsymbol{v}$ could be viewed as the insertion rule of an odd-symmetric, binary interpolating scheme defined by the subdivision mask $\tilde{\boldsymbol{v}}=\left\{\tilde{v}_{i}=\varphi\left(\frac{i}{2}\right), i=1, \ldots, 2 S-1\right\}$ of the form

$$
\tilde{\boldsymbol{v}}=\left\{0, v_{1}, 0, v_{2}, 0, \ldots, 0, v_{\frac{S-1}{2}}, 1, v_{\frac{S+1}{2}}, 0, \ldots, 0, v_{S-2}, 0, v_{S-1}, 0\right\} .
$$

For instance, $\boldsymbol{v}$ could be defined by the coefficients of the insertion rule of the Dubuc-Deslauriers $(2 N)$-point scheme with $N=\frac{S-1}{2}$, given by (see [9])

$$
v_{j+N+1}=\frac{N}{2^{4 N-3}}\left(\begin{array}{c}
2 N-1 \\
N-1
\end{array}\right) \frac{(-1)^{j}}{2 j+1}\left(\begin{array}{c}
2 N-1 \\
N+j
\end{array}\right), \quad j=-N, \ldots, N-1 .
$$

Hence, once $\boldsymbol{v}$ is assigned, we can exploit the equations of the linear system in (3.3) to derive the constraints to be fulfilled by the entries of the even-symmetric subdivision mask $\boldsymbol{a}$ in such a way that its $m$-refinable function $\varphi$ satisfies the properties 
P1) $\operatorname{supp}(\varphi)=[0, S]$;

P2) $\varphi\left(\frac{n}{2}\right)=\tilde{v}_{n}, n=1, \ldots 2 S-1$ or, equivalently, $\varphi\left(n+\frac{S}{2}\right)=\delta_{0, n}, n \in \mathbb{Z}$ and $\varphi(n)=v_{n}, n=1, \ldots, S-1$.

Remark 3.3. It is not difficult to see that, when $m=2$, the equation obtained from 3.2 by setting $i=1$ and assuming $\varphi(0)=0$, reads as

$$
\varphi\left(\frac{1}{2}\right)=a_{0} \varphi(1)
$$

Therefore, excluding the subdivision mask $\boldsymbol{a}=\{1,1\}$ for the trivial case $M=1$, which yields a 2-refinable function $\varphi$ that is the characteristic function of the interval $(0,1]$ and thus verifies $(3.5)$ with the values $\varphi\left(\frac{1}{2}\right)=\varphi(1)=1$, equation (3.5) can never be satisfied when $M>1$. In fact, in light of our assumptions, when $M>1$ neither $a_{0}$ nor $\varphi(1)$ can be zero, whereas $\varphi\left(\frac{1}{2}\right)=0$ is required in order to satisfy (3.4) with $S>1$. Thus, the linear system of equations in (3.2) has no solutions in the case $m=2, M>1$.

In light of Remark 3.3 in what follows we perform all computations with dilation factor $m=4$. In fact, quaternary schemes in addition to allowing for a quicker generation of curves than binary and ternary schemes [14, have still a reasonable cost.

\subsection{Even-symmetric, interpolating 4-refinable functions: a constructive algorithm}

Let $m=4$. Since, according to our assumptions, $M$ must be odd and multiple of 3 , we set $M=$ $2(3 N+1)+1, N \in \mathbb{N}$. In this way $\frac{M}{m-1}=2 N+1$ for all $N \in \mathbb{N}$. Then, we consider the finite mask $\boldsymbol{a}=\left\{a_{0}, \ldots, a_{6 N+3}\right\}$ whose entries satisfy

$$
a_{i}= \begin{cases}b_{3 N+1-i}, & \text { if } 0 \leq i \leq 3 N+1, \\ b_{i-(3 N+2),}, & \text { if } 3 N+2 \leq i \leq 6 N+3,\end{cases}
$$

where $b_{0}, \ldots, b_{3 N+1}$ denote $3 N+2$ unknown weights.

Proposition 3.4. Let $\mathcal{S}_{\boldsymbol{a}}$ be a quaternary subdivision scheme defined by the even-symmetric mask in (3.6). If $\mathcal{S}_{\boldsymbol{a}}$ is interpolatory, then it is a true quaternary scheme and not an iterated, two-step binary scheme.

Proof. The proof is obtained by contradiction. Suppose there exists a symbol $\tilde{A}(z)$ of a convergent, binary scheme $\mathcal{S}_{\tilde{a}}$ such that

$$
A(z)=\tilde{A}(z) \tilde{A}\left(z^{2}\right) .
$$

Since $\operatorname{deg}(A(z))=3(2 N+1)$ and $A(z)$ is even-symmetric, then $\operatorname{deg}(\tilde{A}(z))=2 N+1$ and $\tilde{A}(z)$ is evensymmetric. In light of Remark 3.3, for all $N \in \mathbb{N}$ the subdivision scheme $\mathcal{S}_{\tilde{a}}$ defined by $\tilde{A}(z)$ is not interpolatory. Thus, $\mathcal{S}_{\boldsymbol{a}}$ is also not interpolatory, so contradicting the assumption.

We now provide an algorithm that, for any $N \in \mathbb{N}$, takes as input the values $v_{i}=\varphi(i), i=1, \ldots, 2 N$ and provides as output all the $2 N+1$ equations to be satisfied by the unknowns $b_{0}, \ldots, b_{3 N+1}$ of the subdivision mask $\boldsymbol{a}$ in such a way that, if convergent, it generates a 4-refinable function $\varphi$ satisfying properties P1) and P2) with $S=2 N+1$. The set of $2 N+1$ equations is obtained by suitably cutting the bi-infinite linear system in 3.3 to obtain a finite linear system

$$
\overline{\mathrm{X}} \overline{\boldsymbol{a}}=\overline{\mathbf{y}}
$$

of size $(2 N+1) \times(4 N+2)$ where

$$
\overline{\mathbf{X}}=\left[X_{i, j+1}\right]_{i=1, \ldots, 2 N+1, j=0, \ldots, 4 N+1}, \quad \overline{\boldsymbol{a}}=\left[a_{j}\right]_{j=0, \ldots, 4 N+1}, \quad \overline{\mathbf{y}}=\left[y_{i}\right]_{i=1, \ldots, 2 N+1}
$$


and

$$
\begin{gathered}
X_{i, j+1}= \begin{cases}\varphi(2 i-j), & \text { if } 0<2 i-j \leq \frac{2 N+1}{2} \\
\varphi(2 N+1-2 i+j), & \text { if } \frac{2 N+1}{2}<2 i-j<2 N+1 \\
\text { otherwise } & \text { for } i=1, \ldots, 2 N+1, j=0, \ldots, 4 N+1\end{cases} \\
a_{j}= \begin{cases}b_{3 N+1-j}, & \text { if } 0 \leq j \leq 3 N+1, \\
b_{j-(3 N+2),} & \text { if } 3 N+2 \leq j \leq 4 N+1,\end{cases} \\
y_{i}=\left\{\begin{array}{ll}
\varphi\left(\frac{i}{2}\right), & \text { if } i \in 2 \mathbb{Z}, \\
1, & \text { if } i=2 N+1, \\
0, & \text { otherwise }
\end{array} \text { for } i=1, \ldots, 2 N+1 .\right.
\end{gathered}
$$

Note that, the definition of $\overline{\mathbf{X}}$ takes into account that the function $\varphi$ has support $[0,2 N+1]$ and is symmetric with respect to the center of its support. Thus, the values required as input are indeed $v_{i}=\varphi(i)$, $i=1, \ldots, N$.

Proposition 3.5. The $(2 N+1) \times(4 N+2)$ coefficient matrix $\overline{\mathbf{X}}$ in $(3.8)$ is full rank and the undetermined linear system $\overline{\mathbf{X}} \overline{\boldsymbol{a}}=\overline{\mathbf{y}}$ in (3.7) is consistent.

Proof. The $2 N$ non-zero entries on each row of $\mathbf{X}$ are the entries of $\boldsymbol{v}$ satisfying $v_{i}=\varphi(i), i=1, \ldots, 2 N$. Moreover, the entries of $\mathbf{X}$ satisfy

$$
X_{i+1, j+1}=\varphi(2(i+1)-j)=\varphi(2 i-(j-2))=X_{i, j-1}, \quad \forall i, j \in \mathbb{Z},
$$

namely the non-zero entries of the $(i+1)$-th row of $\mathbf{X}$ are shifted forward by two places with respect to those of the $i$-th row. As a consequence each of the rows of $\overline{\mathbf{X}}$ are linearly independent. Thus $\operatorname{rank}(\overline{\mathbf{X}})=2 N+1$, i.e., the coefficient matrix of the linear system in (3.7) is full rank.

Finally, in light of the fact that $y_{2 i} \neq 0$ for all $i \in \mathbb{Z}$ whereas $y_{2 i+1}=0$ for all $i \neq N$, it follows that

$$
\operatorname{rank}([\overline{\mathbf{X}}, \overline{\mathbf{y}}])=2 N+1
$$

where $[\overline{\mathbf{X}}, \overline{\mathbf{y}}]$ denotes the augmented matrix, i.e., the coefficient matrix $\overline{\mathbf{X}}$ with the column vector $\overline{\mathbf{y}}$ added.

For the sake of clarity, we include two examples of the coefficient matrix $\overline{\mathbf{X}}$ and the corresponding known term $\overline{\mathbf{y}}$ in the cases $N=2$ and $N=3$. Precisely,

- when $N=2$ :

$$
\overline{\mathbf{X}}=\left[\begin{array}{cccccccccc}
\varphi(2) & \varphi(1) & 0 & 0 & 0 & 0 & 0 & 0 & 0 & 0 \\
\varphi(1) & \varphi(2) & \varphi(2) & \varphi(1) & 0 & 0 & 0 & 0 & 0 & 0 \\
0 & 0 & \varphi(1) & \varphi(2) & \varphi(2) & \varphi(1) & 0 & 0 & 0 & 0 \\
0 & 0 & 0 & 0 & \varphi(1) & \varphi(2) & \varphi(2) & \varphi(1) & 0 & 0 \\
0 & 0 & 0 & 0 & 0 & 0 & \varphi(1) & \varphi(2) & \varphi(2) & \varphi(1)
\end{array}\right], \overline{\mathbf{y}}=\left[\begin{array}{c}
0 \\
\varphi(1) \\
0 \\
\varphi(2) \\
1
\end{array}\right]
$$

- when $N=3$ :

$$
\mathbf{\mathbf { X }}=\left[\begin{array}{cccccccccccccc}
\varphi(2) & \varphi(1) & 0 & 0 & 0 & 0 & 0 & 0 & 0 & 0 & 0 & 0 & 0 & 0 \\
\varphi(3) & \varphi(3) & \varphi(2) & \varphi(1) & 0 & 0 & 0 & 0 & 0 & 0 & 0 & 0 & 0 & 0 \\
\varphi(1) & \varphi(2) & \varphi(3) & \varphi(3) & \varphi(2) & \varphi(1) & 0 & 0 & 0 & 0 & 0 & 0 & 0 & 0 \\
0 & 0 & \varphi(1) & \varphi(2) & \varphi(3) & \varphi(3) & \varphi(2) & \varphi(1) & 0 & 0 & 0 & 0 & 0 & 0 \\
0 & 0 & 0 & 0 & \varphi(1) & \varphi(2) & \varphi(3) & \varphi(3) & \varphi(2) & \varphi(1) & 0 & 0 & 0 & 0 \\
0 & 0 & 0 & 0 & 0 & 0 & \varphi(1) & \varphi(2) & \varphi(3) & \varphi(3) & \varphi(2) & \varphi(1) & 0 & 0 \\
0 & 0 & 0 & 0 & 0 & 0 & 0 & 0 & \varphi(1) & \varphi(2) & \varphi(3) & \varphi(3) & \varphi(2) & \varphi(1)
\end{array}\right], \overline{\mathbf{y}}=\left[\begin{array}{c}
0 \\
\varphi(1) \\
0 \\
\varphi(2) \\
0 \\
\varphi(3) \\
1
\end{array}\right] .
$$


The following algorithm summarizes in three steps how to set up a consistent linear system that can yield at least one set of values for the unknowns $b_{0}, \ldots, b_{3 N+1}$ of the subdivision mask $\boldsymbol{a}$ of an even-symmetric, 4-refinable function $\varphi$ satisfying $\mathrm{P} 1$ ) and P2) with $S=2 N+1$.

Algorithm 1 (Constructive algorithm). Select the desired value of $N \in \mathbb{N}$ and provides as input the first $N$ entries $v_{i}=\varphi(i), i=1, \ldots, N$ of the vector $\boldsymbol{v}$ satisfying $v_{2 N+1-i}=v_{i}, i=1, \ldots, N$ and $\sum_{i=1}^{2 N} v_{i}=1$.

- Step 1: Write the $2 N+1$ equations obtained from $\overline{\mathbf{X}} \overline{\boldsymbol{a}}-\overline{\mathbf{y}}=\mathbf{0}$ in (3.7).

- Step 2: Add to the $2 N+1$ equations from Step 1 the adequate number of consistent equations (not larger than $N+1)$ to be satisfied by $\boldsymbol{a}$ in such a way that $\mathcal{S}_{\boldsymbol{a}}$ generates polynomials of a certain degree $d$. In this regard we recall that generation of polynomials of degree $d$ is a necessary condition for $C^{d}$ smoothness of the function $\varphi$ (see Appendix A) and its fulfillment requires the input vector $\boldsymbol{v}$ to collect the samples at integers of an interpolating function reproducing $\Pi_{d}$.

- Step 3: Solve the linear system obtained by assembling the equations obtained in Step 1 and 2 in order to get the even-symmetric mask $\boldsymbol{a}$ in (3.6) for the desired quaternary interpolating subdivision scheme $\mathcal{S}_{a}$.

In the next two Subsections we illustrate the outcomes of Algorithm 1 in the case $S=\frac{M}{m-1}=5$ (i.e., $N=2$ ) and $S=\frac{M}{m-1}=7$ (i.e., $N=3$ ).

3.2.1. Example 1: even-symmetric quaternary counterpart of the interpolating DD 4-point scheme

We denote by $\varphi_{D D 4}$ the interpolating 2-refinable function (shown in Fig. 1 left) that is obtained from the binary Dubuc-Deslauriers 4-point scheme (DD4 for short) [8, 9] and satisfies the conditions

- $\operatorname{supp}\left(\varphi_{D D 4}\right)=[0,6]$ and $\varphi(x)=\varphi(6-x)$ for all $x \in \mathbb{R}$;

- $\varphi_{D D 4}(n+3)=\delta_{0, n}, \quad n \in \mathbb{Z}$;

- $\varphi_{D D 4}\left(n+\frac{3}{2}\right)= \begin{cases}0, & n=-1,4 \\ \frac{3}{16} \frac{(-1)^{n}}{2 n-3}\left(\begin{array}{l}3 \\ n\end{array}\right), & n=0, \ldots, 3 .\end{cases}$
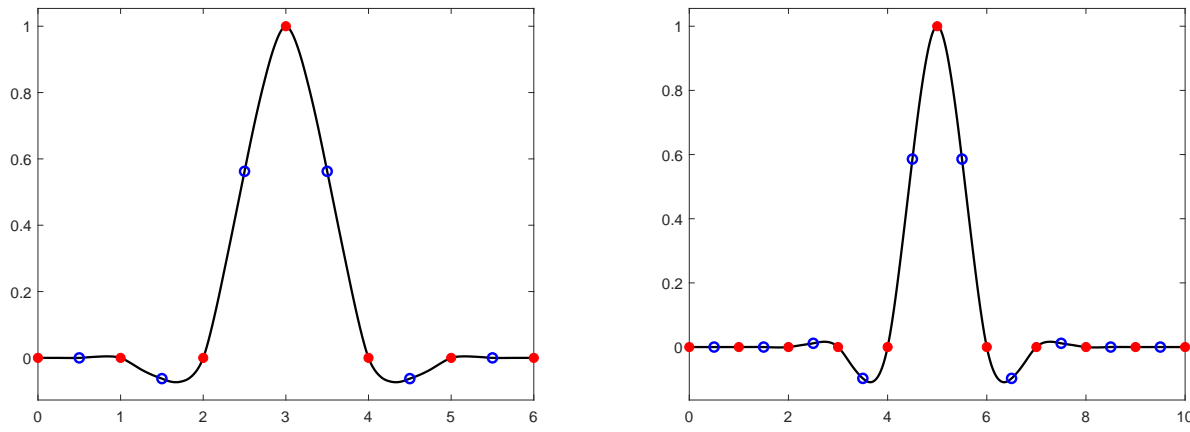

Figure 1: Left: $C^{1}$ interpolating 2-refinable function provided by the binary DD 4-point scheme. Right: $C^{2}$ interpolating 2-refinable function provided by the binary DD 6-point scheme.

When $\frac{M}{m-1}=5$, i.e., $N=2$, we look for a subdivision mask of the form

$$
\boldsymbol{a}=\left\{a_{i}\right\}_{i=0}^{15}=\left\{b_{7}, b_{6}, b_{5}, b_{4}, b_{3}, b_{2}, b_{1}, b_{0}, b_{0}, b_{1}, b_{2}, b_{3}, b_{4}, b_{5}, b_{6}, b_{7}\right\}
$$


that is associated to a 4-refinable function $\varphi$ having support $[0,5]$. In order to apply Algorithm 1 , we require $\varphi$ to meet the values

$$
\left\{\varphi\left(\frac{n}{2}\right)\right\}_{n=1, \ldots, 9}=\left\{0,-\frac{1}{16}, 0, \frac{9}{16}, 1, \frac{9}{16}, 0,-\frac{1}{16}, 0\right\}
$$

attained at the half-integers by the interpolating 2-refinable function provided by the binary DD 4-point scheme (see Fig. 1 left). With such an input, Step 1 of Algorithm 1 provides the following constraints

$$
\left\{\begin{array}{l}
\frac{9}{16} b_{7}-\frac{1}{16} b_{6}=0 \\
-\frac{1}{16} b_{7}+\frac{9}{16} b_{6}+\frac{9}{16} b_{5}-\frac{1}{16} b_{4}=-\frac{1}{16} \\
-\frac{1}{16} b_{5}+\frac{9}{16} b_{4}+\frac{9}{16} b_{3}-\frac{1}{16} b_{2}=0 \\
-\frac{1}{16} b_{3}+\frac{9}{16} b_{2}+\frac{9}{16} b_{1}-\frac{1}{16} b_{0}=\frac{9}{16} \\
-\frac{1}{16} b_{1}+\frac{9}{16} b_{0}=\frac{1}{2}
\end{array}\right.
$$

to be satisfied by the entries of the subdivision mask 3.9 .

Recalling that $\varphi_{D D 4}$ reproduces $\Pi_{3}$ with the shortest possible support [8] and pointing out that $\operatorname{supp}(\varphi)=$ $[0,5] \subset \operatorname{supp}\left(\varphi_{D D 4}\right)$, we can infer that there is no suitable choice of the mask entries to achieve reproduction of $\Pi_{3}$. Thus, we perform Step 2 by requiring $\Pi_{2}$-reproduction, i.e., the fulfillment of conditions (2.2) with $d=2$ and $\tau_{\boldsymbol{a}}=\frac{15}{2}$. What we get by the solution of the linear system in Step 3 is a one-parameter mask with coefficients

$$
\begin{array}{llll}
b_{7}=\frac{\alpha}{16}, & b_{6}=\frac{9}{16} \alpha, & b_{5}=-\frac{9}{16} \alpha-\frac{15}{128}, & b_{4}=-\frac{\alpha}{16}-\frac{7}{128}, \\
b_{3}=\frac{9}{128}-\frac{3}{16} \alpha, & b_{2}=\frac{33}{128}-\frac{27}{16} \alpha, & b_{1}=\frac{27}{16} \alpha+\frac{55}{64}, & b_{0}=\frac{3}{16} \alpha+\frac{63}{64}
\end{array} \quad \alpha \in \mathbb{R} .
$$

In light of 3.10$)$, we call the subdivision scheme $\mathcal{S}_{\boldsymbol{a}}$ defined by $3.9-(3.11)$ the even-symmetric quaternary counterpart of the interpolating DD 4-point scheme.

Proposition 3.6. The basic limit function $\varphi$ of the even-symmetric quaternary subdivision scheme with mask 3.9)-3.11) has symbol

$$
A(z)=\frac{1}{128}\left(z^{3}+z^{2}+z+1\right)^{3}\left(8 \alpha z^{6}+48 \alpha z^{5}-3(88 \alpha+5) z^{4}+2(208 \alpha+19) z^{3}-3(88 \alpha+5) z^{2}+48 \alpha z+8 \alpha\right)
$$

and satisfies the following properties:

i) $\operatorname{supp}(\varphi)=[0,5]$ and $\varphi(x)=\varphi(5-x)$ for all $x \in \mathbb{R}$;

ii) $\varphi\left(n+\frac{5}{2}\right)=\delta_{0, n}, \quad n \in \mathbb{Z}$;

iii) $\varphi(n+1)=\frac{3}{16} \frac{(-1)^{n}}{2 n-3}\left(\begin{array}{l}3 \\ n\end{array}\right), \quad n=0, \ldots, 3$;

iv) $\{\varphi(\cdot-i)\}_{i \in \mathbb{Z}}$ reproduces $\Pi_{2}$ for all values of $\alpha$;

v) $\varphi \in C^{2}(\mathbb{R})$ for all $\alpha \in\left[-0.0862,-\frac{15}{208}\right) \approx[-0.0862,-0.0721)$.

Proof: Properties $i), i i), i i i), i v$ ) follow by construction. Property $v$ ) follows from the Hölder regularity analysis relying on the computation of the joint spectral radius of the set of matrices $\left\{D_{0}^{[3]}, D_{1}^{[3]}, D_{2}^{[3]}, D_{3}^{[3]}\right\}$ (see Appendix A with

$$
D_{0}^{[3]}=\left[\begin{array}{cccccc}
d_{0}^{[3]} & d_{2}^{[3]} & 0 & 0 & 0 & 0 \\
0 & d_{3}^{[3]} & 0 & 0 & 0 & 0 \\
0 & d_{2}^{[3]} & d_{0}^{[3]} & 0 & 0 & 0 \\
0 & d_{1}^{[3]} & d_{1}^{[3]} & 0 & 0 & 0 \\
0 & d_{0}^{[3]} & d_{2}^{[3]} & 0 & 0 & 0 \\
0 & 0 & d_{3}^{[3]} & 0 & 0 & 0
\end{array}\right], \quad D_{1}^{[3]}=\left[\begin{array}{cccccc}
0 & d_{3}^{[3]} & 0 & 0 & 0 & 0 \\
0 & d_{2}^{[3]} & d_{0}^{[3]} & 0 & 0 & 0 \\
0 & d_{1}^{[3]} & d_{1}^{[3]} & 0 & 0 & 0 \\
0 & d_{0}^{[3]} & d_{2}^{[3]} & 0 & 0 & 0 \\
0 & 0 & d_{3}^{[3]} & 0 & 0 & 0 \\
0 & 0 & d_{2}^{[3]} & d_{0}^{[3]} & 0 & 0
\end{array}\right],
$$




$$
D_{2}^{[3]}=\left[\begin{array}{cccccc}
0 & d_{2}^{[3]} & d_{0}^{[3]} & 0 & 0 & 0 \\
0 & d_{1}^{[3]} & d_{1}^{3]} & 0 & 0 & 0 \\
0 & d_{0}^{[3]} & d_{2}^{[3]} & 0 & 0 & 0 \\
0 & 0 & d_{3}^{[3]} & 0 & 0 & 0 \\
0 & 0 & d_{2}^{[3]} & d_{0}^{[3]} & 0 & 0 \\
0 & 0 & d_{1}^{[3]} & d_{1}^{[3]} & 0 & 0
\end{array}\right], \quad D_{3}^{[3]}=\left[\begin{array}{cccccc}
0 & d_{1}^{[3]} & d_{1}^{[3]} & 0 & 0 & 0 \\
0 & d_{0}^{[3]} & d_{2}^{[3]} & 0 & 0 & 0 \\
0 & 0 & d_{3}^{[3]} & 0 & 0 & 0 \\
0 & 0 & d_{2}^{[3]} & d_{0}^{[3]} & 0 & 0 \\
0 & 0 & d_{1}^{[3]} & d_{1}^{[3]} & 0 & 0 \\
0 & 0 & d_{0}^{[3]} & d_{2}^{[3]} & 0 & 0
\end{array}\right]
$$

that are associated with the symbol

$$
D^{[3]}(z)=\frac{4^{3} A(z)}{\left(z^{2}+1\right)^{3}(z+1)^{3}}=d_{0}^{[3]} z^{6}+d_{1}^{[3]} z^{5}+d_{2}^{[3]} z^{4}+d_{3}^{[3]} z^{3}+d_{2}^{[3]} z^{2}+d_{1}^{[3]} z+d_{0}^{[3]}
$$

of the 3 rd-order difference scheme of $\mathcal{S}_{\boldsymbol{a}}$ defined by the coefficients

$$
d_{0}^{[3]}=4 \alpha, \quad d_{1}^{[3]}=24 \alpha, \quad d_{2}^{[3]}=-132 \alpha-\frac{15}{2}, \quad d_{3}^{[3]}=208 \alpha+19 .
$$

By using the modified invariant polytope algorithm proposed in 17] we can compute the exact value of the joint spectral radius $\rho$, which turns out to satisfy the constraint $\rho<4$ for all $\alpha \in\left[-0.0862,-\frac{15}{208}\right)$. Thus, for all these values of $\alpha$ the Hölder regularity of the subdivision scheme $\mathcal{S}_{\boldsymbol{a}}$ is $\nu=3-\log _{4}(\rho)>2$ (see Fig. 2 -right).

Remark 3.7. $\mathcal{S}_{\boldsymbol{a}}$ belongs to the family of even-symmetric de Rham-type quaternary schemes (see [6]) since its symbol $A(z)$ can be written as the product of the odd sub-symbol $C_{\text {odd }}(z)=\frac{1}{4}(z+1)\left(z^{2}+1\right)$ of a binary subdivision scheme $\mathcal{S}_{c}$ defined by $C(z)=\frac{1}{4}(z+1)^{2}\left(z^{4}+2(2 \sigma-1) z^{3}+4(1-2 \sigma) z^{2}+2(2 \sigma-1) z+1\right), \sigma \in \mathbb{R}$ and

$B(z)=\frac{1}{32}\left(z^{3}+z^{2}+z+1\right)^{2}\left(8 \alpha z^{6}+48 \alpha z^{5}-3(88 \alpha+5) z^{4}+2(208 \alpha+19) z^{3}-3(88 \alpha+5) z^{2}+48 \alpha z+8 \alpha\right)$.

The latter is the symbol of an odd-symmetric quaternary scheme $\mathcal{S}_{\boldsymbol{b}}$ that is $C^{1}$-convergent for all $\alpha \in$ $\left[-0.0862,-\frac{15}{208}\right)$ and $\Pi_{1}$-reproducing. In contrast to [6] where only examples of even-symmetric approximating de Rham-type schemes are presented, $A(z)$ is the symbol of the first example of interpolating de Rham-type scheme. Note that, $\mathcal{S}_{\boldsymbol{a}}$ is smoother than the odd-symmetric scheme it is built upon, since $C_{\text {odd }}(z)$ is a smoothing factor for $\mathcal{S}_{\boldsymbol{b}}$.

Remark 3.8. $\mathcal{S}_{a}$ provides a parameter-dependent, interpolating 4-refinable function with the properties of $\Pi_{2}$-reproduction, $C^{2}$-smoothness and support width 5 exactly as the ternary 4-point scheme defined by the interpolating symbol [19]

$$
\frac{1}{9}\left(z^{2}+z+1\right)^{3}\left(9 \gamma z^{4}-(36 \gamma+1) z^{3}+(54 \gamma+3) z^{2}-(36 \gamma+1) z+9 \gamma\right), \quad \gamma \in \mathbb{R} .
$$

Indeed, the ternary scheme in 3.13 yields a basic limit function $\varphi$ characterized by:

- $\operatorname{supp}(\varphi)=[0,5]$ and $\varphi(x)=\varphi(5-x)$ for all $x \in \mathbb{R}$;

- $\varphi\left(n+\frac{5}{2}\right)=\delta_{0, n}, \quad n \in \mathbb{Z}$;

- $\{\varphi(\cdot-i)\}_{i \in \mathbb{Z}}$ reproduces $\Pi_{2}$ for all values of $\gamma$;

- $\varphi \in C^{2}(\mathbb{R})$ for all $\gamma \in\left(-\frac{2}{45},-\frac{1}{27}\right) \approx(-0.0444,-0.0370)$. 
Figure 3 shows a graphical illustration of the interpolating refinable functions with symbols $(3.12)$ and (3.13). Note that we do not center them at the axes origin (as it is consuetude), but we plot them in [0,5], to be consistent with the notation previously used to denote the support interval. Although their shape is very similar, it is worthwhile to point out the remarkable differences between the underlying subdivision schemes. In particular, the basic limit function of the subdivision scheme with symbol (3.13) is associated with the standard type of odd-symmetric mask

$$
\left\{\gamma,-\gamma-\frac{1}{9}, 0, \frac{2}{9}-3 \gamma, 3 \gamma+\frac{8}{9}, 1,3 \gamma+\frac{8}{9}, \frac{2}{9}-3 \gamma, 0,-\gamma-\frac{1}{9}, \gamma\right\}
$$

Since its length is 11 and the arity is 3 , the shift parameter is integer and precisely $\tau_{\boldsymbol{a}}=5$. Differently, the basic limit function of the subdivision scheme with symbol (3.12) is associated with the new type of evensymmetric mask in (3.9). Since its length is 16 and the arity is 4, its shift parameter is not integer, but given by $\tau_{\boldsymbol{a}}=\frac{15}{2}$.
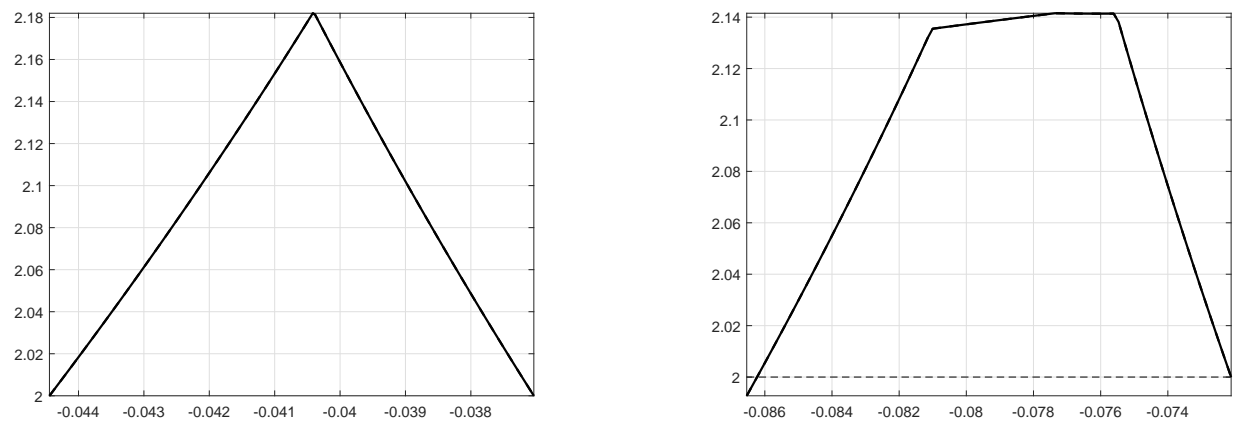

Figure 2: Left: Hölder regularity $\nu$ of the odd-symmetric subdivision scheme with mask in (3.14) for $\gamma \in\left(-\frac{2}{45},-\frac{1}{27}\right)$. Right: Hölder regularity $\nu$ of the even-symmetric subdivision scheme with mask in 3.9 - 3.11 for $\alpha \in\left(-\frac{9}{104},-\frac{15}{208}\right)$.
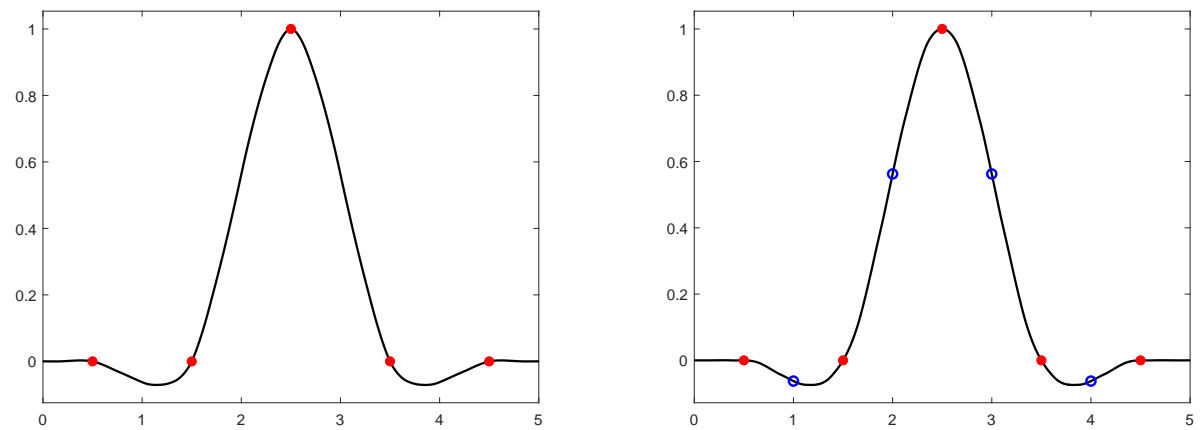

Figure 3: Left: $C^{2}$ interpolating 2-refinable function provided by the odd-symmetric ternary $(m=3) 4$-point scheme having symbol in 3.13 with $\gamma=-0.0404$ (such that the Hölder regularity reaches the maximum value $\nu=2.18$ highlighted in Fig. 2.left). Right: $C^{2}$ interpolating 4-refinable function provided by the even-symmetric quaternary $(m=4)$ scheme having symbol in 3.12 with $\alpha=-0.0773$ (such that the Hölder regularity reaches the maximum value $\nu=2.14$ highlighted in Fig. 2 2 right). Blue circles and red bullets mark the interpolated values assigned by the binary mask in 3.10 .

Figure 4 shows an application example of the new even-symmetric, quaternary subdivision scheme and compares it with the existing ternary, odd-symmetric subdivision scheme featured by the same properties. 


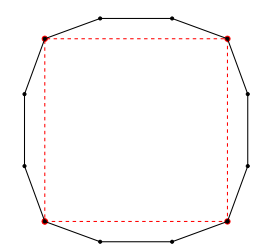

(a)

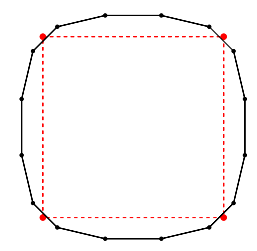

(b)

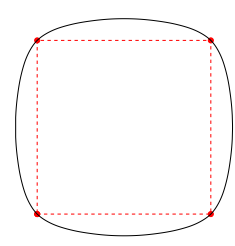

(a)

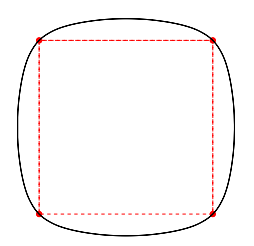

(b)

Figure 4: First column: given data and first step of subdivision; second column: given data and limit curve. In (a) the $C^{2}$ ternary 4-point scheme in 3.13 with $\gamma=-0.0404$; in (b) the $C^{2}$ even-symmetric quaternary 4-point scheme in 3.12 with $\alpha=-0.0773$.

\subsubsection{Example 2: even-symmetric quaternary counterpart of the parameter-dependent interpolating 6-point scheme}

Following the lines of reasoning previously used in Subsection 3.2.1, that is, deriving first the conditions on the mask coefficients provided by Step 1 of Algorithm 1, and successively requiring the subdivision mask to meet the desired degree of polynomial reproduction (according to Step 2), we can also construct evensymmetric interpolating subdivision schemes for the cases $\frac{M}{m-1}=2 N+1, N \geq 3$.

For instance, when $\frac{M}{m-1}=7$ (i.e., $N=3$ ), if we take as input the values

$$
\left\{\varphi\left(\frac{n}{2}\right)\right\}_{n=1, \ldots, 13}=\left\{0, \theta, 0,-\left(3 \theta+\frac{1}{16}\right), 0,\left(2 \theta+\frac{9}{16}\right), 1,\left(2 \theta+\frac{9}{16}\right), 0,-\left(3 \theta+\frac{1}{16}\right), 0, \theta, 0\right\}
$$

attained at the half-integers by the interpolating 2-refinable function provided by the parameter-dependent, binary, 6-point scheme in [13, the output of Step 1 of Algorithm 1 are the constraints

$$
\left\{\begin{array}{l}
b_{9} \theta-b_{10}\left(3 \theta+\frac{1}{16}\right)=0 \\
b_{7} \theta-b_{8}\left(3 \theta+\frac{1}{16}\right)+\left(b_{9}+b_{10}\right)\left(2 \theta+\frac{9}{16}\right)=\theta \\
\left(b_{5}+b_{10}\right) \theta-\left(b_{6}+b_{9}\right)\left(3 \theta+\frac{1}{16}\right)+\left(b_{7}+b_{8}\right)\left(2 \theta+\frac{9}{16}\right)=0 \\
\left(b_{3}+b_{8}\right) \theta-\left(b_{4}+b_{7}\right)\left(3 \theta+\frac{1}{16}\right)+\left(b_{5}+b_{6}\right)\left(2 \theta+\frac{9}{16}\right)=-\left(3 \theta+\frac{1}{16}\right), \\
\left(b_{1}+b_{6}\right) \theta-\left(b_{2}+b_{5}\right)\left(3 \theta+\frac{1}{16}\right)+\left(b_{3}+b_{4}\right)\left(2 \theta+\frac{9}{16}\right)=0 \\
\left(b_{0}+b_{4}\right) \theta-\left(b_{0}+b_{3}\right)\left(3 \theta+\frac{1}{16}\right)+\left(b_{1}+b_{2}\right)\left(2 \theta+\frac{9}{16}\right)=\left(2 \theta+\frac{9}{16}\right), \\
b_{0}\left(2 \theta+\frac{9}{16}\right)-b_{1}\left(3 \theta+\frac{1}{16}\right)+b_{2} \theta=\frac{1}{2} .
\end{array}\right.
$$

The 7 equations in $(3.16$ have to be fulfilled by the entries of the subdivision mask

$$
\boldsymbol{a}=\left\{a_{i}\right\}_{i=0}^{21}=\left\{b_{10}, b_{9}, b_{8}, b_{7}, b_{6}, b_{5}, b_{4}, b_{3}, b_{2}, b_{1}, b_{0}, b_{0}, b_{1}, b_{2}, b_{3}, b_{4}, b_{5}, b_{6}, b_{7}, b_{8}, b_{9}, b_{10}\right\}
$$

in order to get a family of 4-refinable functions satisfying the properties

i) $\operatorname{supp}(\varphi)=[0,7]$ and $\varphi(x)=\varphi(7-x)$ for all $x \in \mathbb{R}$;

ii) $\varphi\left(n+\frac{7}{2}\right)=\delta_{0, n}, \quad n \in \mathbb{Z}$;

iii) $\varphi(n+1)= \begin{cases}\theta, & n=0,5, \\ \frac{(-1)^{n-1}}{2 n-5}\left(\left(\frac{3}{16}-16 \theta\right)\left(\begin{array}{c}3 \\ n-1\end{array}\right)+5 \theta\left(\begin{array}{c}5 \\ n\end{array}\right)\right), & n=1, \ldots, 4,\end{cases}$

which allow us to call it the even-symmetric quaternary counterpart of the parameter-dependent interpolating 6-point scheme. Since the interpolating 2-refinable function obtained from the 6-point scheme with mask in 3.15 reproduces $\Pi_{3}$ for all values of $\theta \in \mathbb{R}$, according to Step 2 of Algorithm 1 , we can also require 
that the family of even-symmetric interpolating 4-refinable functions has the property of cubic polynomial reproduction. Doing this, the solution to the linear system in Step 3 yields for (3.17) a one-parameter mask with

$$
\begin{array}{llll}
b_{10}=-\frac{3}{256} \lambda, & b_{9}=-\frac{9}{256} \lambda-\mu+\frac{127}{8192}, & b_{8}=-\frac{3}{64} \lambda-\eta, & b_{7}=-\frac{3}{64} \lambda-\eta-\frac{21}{1024}, \\
b_{6}=\frac{3}{256} \lambda-\mu-\frac{313}{8192}, & b_{5}=\frac{33}{256} \lambda+4 \mu-\frac{257}{2048}, & b_{4}=\frac{3}{16} \lambda+4 \eta-\frac{35}{1024}, & b_{3}=\frac{3}{16} \lambda+4 \eta+\frac{135}{1024}, \\
b_{2}=\frac{9}{128} \lambda+4 \mu+\frac{731}{2048}, & b_{1}=-\frac{21}{128} \lambda-6 \mu+\frac{3241}{4096}, & b_{0}=-\frac{9}{32} \lambda-6 \eta+\frac{945}{1024}, &
\end{array}
$$

and

$$
\lambda=-\frac{\theta\left(520192 \theta^{2}+152160 \theta+67\right)}{12(32 \theta+5)(64 \theta+1)}, \quad \mu=\frac{1203-62752 \theta}{2^{14}(32 \theta+5)(64 \theta+1)}, \quad \eta=\frac{8278016 \theta^{2}-48992 \theta-603}{2^{14}(32 \theta+5)(64 \theta+1)} .
$$

The associated symbol is

$$
A(z)=\frac{\left(z^{2}+1\right)^{4}(z+1)^{5}\left(c_{0} z^{8}+c_{1} z^{7}+c_{2} z^{6}+\left(c_{3}-c_{1}\right) z^{5}+\left(c_{1}-c_{4}\right) z^{4}+\left(c_{3}-c_{1}\right) z^{3}+c_{2} z^{2}+c_{1} z+c_{0}\right)}{2^{1} 4(32 \theta+5)(64 \theta+1)}
$$

where

$$
\begin{gathered}
c_{0}=16 \theta\left(520192 \theta^{2}+152160 \theta+67\right), \quad c_{1}=(1-32 \theta)\left(520192 \theta^{2}+152160 \theta+67\right), \\
c_{2}=-4\left(2719744 \theta^{2}+177952 \theta-67\right), \quad c_{3}=8\left(4833280 \theta^{2}+188960 \theta-411\right), \\
c_{4}=55832576 \theta^{2}+1706848 \theta-6613 .
\end{gathered}
$$

Analyzing its Hölder regularity via the analysis technique described in Appendix A we find that the corresponding interpolating 4-refinable function $\varphi$ is $C^{2}$-continuous for all $\theta \in[-0.00111,0.00723]$ (see Fig. 5-left).

We point out that, when $\theta=0$, the input values in 3.15 coincide with those in $(3.10)$. However, in this case, the subdivision scheme reproduces $\Pi_{3}$ (instead of $\Pi_{2}$ only) and is also $C^{2}$-continuous. Differently, when $\theta=\frac{3}{256}$, the input values in (3.15) are exactly those of the Dubuc-Deslauriers 6-point scheme which reproduces $\Pi_{5}$, but the subdivision scheme in (3.17) with $M=21$ reproduces $\Pi_{4}$ only. In order to reach the property of $\Pi_{5}$-reproduction we should consider a subdivision mask $\boldsymbol{a}$ with more entries. In fact, the interpolating 2-refinable function obtained from the DD 6-point scheme reproduces $\Pi_{5}$ with the shortest possible support (whose width is exactly 10, see Fig. 1 right), and all the 4-refinable functions in our family have support width smaller than 10 (see i)). Figure 5 -right shows the 4-refinable function obtained with the choice of $\theta$ yielding the highest Hölder regularity (see Fig. 5-left).
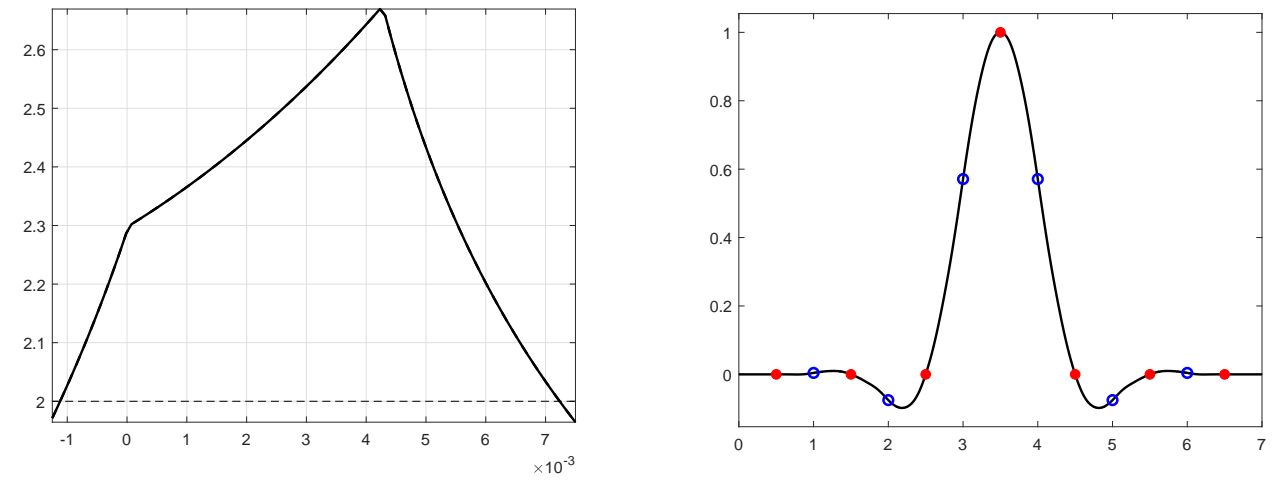

Figure 5: Left: Hölder regularity $\nu$ of the even-symmetric subdivision scheme with mask in (3.17)- $\sqrt{3.18}$ for $\theta \in\left(-\frac{1}{800}, \frac{3}{400}\right)$. Right: $C^{2}$ interpolating 4-refinable function provided by the quaternary $(m=4)$ scheme having mask in $(3.17)-(3.18)$ with $\theta=0.0042$ (such that the Hölder regularity reaches the maximum value $\nu=2.67$ ). Blue circles and red bullets mark the interpolated values assigned by the binary mask in 3.15. 


\section{Closing remarks}

This paper addresses the construction of a novel generation of compactly supported interpolating refinable functions and offers an insight of interpolatory schemes that is lacking in existing literature. In particular, it shows that interpolating refinable functions with even dilation factor $m>2$ can be obtained as basic limit functions of even-symmetric subdivision schemes. A constructive algorithm for generating subdivision schemes belonging to the new class is proposed, and two application examples are illustrated. From the point of view of applications, the new class of interpolatory subdivision schemes can be used to generate a rich family of even-symmetric interpolating refinable functions, that allow the user to meet various demands for balancing polynomial reproduction, regularity and support size.

\section{Acknowledgements}

The author would like to thank Malcolm Sabin for having suggested the open problem treated in this paper. Special thanks also go to the referees for their constructive comments.

\section{References}

[1] C. Beccari, G. Casciola, L. Romani, Shape controlled interpolatory ternary subdivision. Appl. Math. Comput. 215 (2009) 916-927.

[2] C. Beccari, G. Casciola, L. Romani, A unified framework for interpolating and approximating univariate subdivision. Appl. Math. Comput. 216 (2010) 1169-1180.

[3] M. Charina, Finiteness conjecture and subdivision, Appl. Comput. Harmon. Anal. 36 (2014) $522-526$.

[4] D.R. Chen, R.Q. Jia, S.D. Riemenschneider, Convergence of vector subdivision schemes in Sobolev spaces. Appl. Comput. Harmon. Anal. 12 (2002) 128-149.

[5] C. Conti, K. Hormann, Polynomial reproduction for univariate subdivision schemes of any arity. J. Approx. Theory 163 (2011) 413-437.

[6] C. Conti, L. Romani, Dual univariate m-ary subdivision schemes of de Rham-type. J. Math. Anal. Appl. 407(2) (2013) $443-456$.

[7] I. Daubechies, J.C. Lagarias, Sets of matrices all infinite products of which converge. Linear Algebra Appl. 161 (1992) $227-263$.

[8] G. Deslauriers, S. Dubuc, Symmetric iterative interpolation processes. Constr. Approx. 5 (1989) $49-68$.

[9] J.M. de Villiers, K.M. Goosen, B.M. Herbst, Dubuc-Deslauriers subdivision for finite sequences and interpolation wavelets on an interval. SIAM J. Math. Anal. 35 (2003) 423-452.

[10] N. Dyn, Subdivision schemes in computer-aided geometric design. In: W. Light (Ed.), Advances in Numerical Analysis, vol. II, Oxford University Press, New York, 1992, pp. 36-10.

[11] N. Dyn, Interpolatory subdivision schemes. In: A. Iske, E. Quak, M.S. Floater (Eds.), Tutorials on Multiresolution in Geometric Modelling - Mathematics and Visualization. Springer, Berlin, Heidelberg, 2002, pp. 25-50.

[12] N. Dyn, D. Levin, Subdivision schemes in geometric modeling. In: Acta Numer., Cambridge University Press, Cambridge, 2002, pp. 73-144.

[13] N. Dyn, D. Levin, J.A. Gregory, A Butterfly subdivision scheme for surface interpolation with tension control. ACM Transactions on Graphics 9 (1990) 160-169.

[14] S. Hashmi, G. Mustafa, Estimating error bounds for quaternary subdivision schemes. J. Math. Anal. Appl. 358 (2009) 159-167.

[15] K.P. Ko, B.G. Lee, G.J. Yoon, A study on the mask of interpolatory symmetric subdivision schemes. Appl. Math. Comput. $187(2)$ (2007) 609-621.

[16] B.-G. Lee, Y.J. Lee, J. Yoon, Stationary binary subdivision schemes using radial basis function interpolation. Adv. Comput. Math. 25 (2006) 57-72.

[17] T. Mejstrik, Joint spectral radius and subdivision schemes. Doctoral Thesis in Mathematics, University of Vienna, 2019 (http://www.tommsch.com/misc/diss.pdf)

[18] G. Muntingh, Symbols and exact regularity of symmetric pseudo-splines of any arity. BIT Numerical Mathematics 57(3) (2017) 867-900

[19] P. Novara, L. Romani, Complete characterization of the regions of $C^{2}$ and $C^{3}$ convergence of combined ternary 4-point subdivision schemes. Appl. Math. Letters 62 (2016) 84-91.

[20] V. Protasov, Spectral factorizations of 2-block Toeplitz matrices and refinement equations. St. Petersburg Math. J. 18(4) (2007), 607-646.

[21] O. Rioul, Simple regularity criteria for subdivision schemes. SIAM J. Math Anal. 23(6) (1992), 1544-1576.

[22] G.C. Rota, W.G. Strang, A note on the joint spectral radius. Indag. Math. 22 (1960) 379-381.

[23] L. Zhang, H. Ma, S. Tang, J. Tan, A combined approximating and interpolating ternary 4-point subdivision scheme. J. Comput. Appl. Math. 349 (2019) 563-578.

[24] B. Zhang, H. Zheng, W. Song, Z. Lin, J. Zhou, Interpolatory subdivision schemes with the optimal approximation order. Appl. Math. Comput. 347 (2019) 1-14. 


\section{Appendix A. A well-established smoothness analysis tool for univariate schemes of arity $m$}

Let $A(z)=\left(\frac{1-z^{m}}{m(1-z)}\right)^{r+1} D^{[r+1]}(z)$ be the subdivision symbol of a univariate scheme of arity $m$, where $D^{[r+1]}(z)$ denotes the symbol of the $(r+1)$-th order difference scheme with mask $\boldsymbol{d}^{[r+1]}=\left\{d_{i}^{[r+1]}, i \in \mathbb{Z}\right\}$. A first approach to investigate the smoothness of the scheme $\mathcal{S}_{\boldsymbol{a}}$ consists in studying the contractivity of the scheme $\mathcal{S}_{\boldsymbol{d}^{[r+1]}}$. This is done by computing the symbol $\tilde{D}(z):=\frac{1}{m} D^{[r+1]}(z)=\sum_{i \in \mathbb{Z}} \tilde{d}_{i} z^{i}$ and checking the existence of $k \in \mathbb{N}$ such that

$$
\left\|\mathcal{S}_{\tilde{d}}^{k}\right\|_{\infty}<1
$$

for

$$
\left\|\mathcal{S}_{\tilde{d}}^{k}\right\|_{\infty}:=\max \left\{\sum_{j \in \mathbb{Z}}\left|\tilde{d}_{i+m^{k} j}^{k]}\right|: i=0,1, \ldots, m^{k}-1\right\}
$$

and

$$
\tilde{d}_{i}^{[1]} \equiv \tilde{d}_{i}, \forall i \in \mathbb{Z}, \quad \tilde{D}^{[k]}(z)=\prod_{h=0}^{k-1} \tilde{D}\left(z^{m^{h}}\right)=: \sum_{i \in \mathbb{Z}} \tilde{d}_{i}^{[k]} z^{i} .
$$

The condition in (A.1) is a sufficient condition for $C^{r}$ continuity of the limit curves produced by the scheme $\mathcal{S}_{a}$ (see, e.g., [12, Section 4.2]).

Necessary and sufficient conditions for $C^{r}$ convergence of $\mathcal{S}_{\boldsymbol{a}}$ can be obtained by exploiting the joint spectral radius approach introduced in [7. In particular, the analysis of an arbitrary subdivision scheme of arity $m$ can be performed by applying the following known result, which can be found in full generality in 4, Theorem 4.1].

Proposition A.1. Assume that $d_{i}^{[r+1]}=0$ if $i<1-L$ or $i>n-L$, with $L, n \in \mathbb{N}$ and let

$$
\begin{aligned}
\rho & :=\rho\left(D_{0}^{[r+1]}, D_{1}^{[r+1]}, \ldots, D_{m-1}^{[r+1]}\right) \\
& =\lim _{p \rightarrow \infty}\left(\max \left\{\left\|D_{\epsilon_{p}}^{[r+1]} \cdots D_{\epsilon_{2}}^{[r+1]} D_{\epsilon_{1}}^{[r+1]}\right\|_{\infty}^{1 / p}: \epsilon_{i} \in\{0,1, \ldots, m-1\}, i=1, \ldots, p\right\}\right)
\end{aligned}
$$

denote the joint spectral radius (JSR) (see [22]) of the set $\left\{D_{0}^{[r+1]}, D_{1}^{[r+1]}, \ldots, D_{m-1}^{[r+1]}\right\}$ of $n \times n$ matrices given by

$$
D_{\epsilon}^{[r+1]}=\left(d_{n+i-m j+\epsilon}^{[r+1]}\right)_{i, j=0, \ldots, n-1}, \quad \epsilon=0,1, \ldots, m-1
$$

(see, e.g., [3, 7, 20, 21]). The subdivision scheme $\mathcal{S}_{\boldsymbol{a}}$ is $C^{r}$ convergent if and only if it generates the space $\Pi_{r}$ of polynomials up to degree $r$, i.e.,

$$
A^{(i)}\left(e^{\frac{2 \pi \mathrm{i}}{m} j}\right)=0 \quad \text { for all } j=1, \ldots, m-1 \quad \text { and } \quad i=0, \ldots, r,
$$

and $\rho<m$.

Remark A.2. Since the Hölder regularity of the subdivision scheme $\mathcal{S}_{\boldsymbol{a}}$ is $\nu=r+1-\log _{m}(\rho)$, we conclude by pointing out that, if $\rho<m$, then $\nu>r$, i.e. the subdivision scheme $\mathcal{S}_{\boldsymbol{a}}$ has integer smoothness $C^{r}$. 